\title{
S-1 Based Doublet as an Adjuvant Chemotherapy for Curatively Resected Stage III Gastric Cancer: Results from the Randomized Phase III POST Trial
}

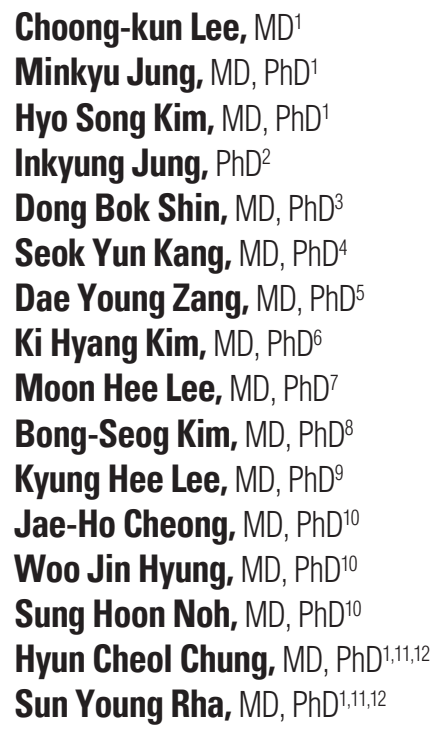

${ }^{*}$ A list author's affiliations appears at the end of the paper.

\section{Purpose}

We conducted a randomized, multicenter, phase III trial to compare S-1 plus docetaxel (DS) with S-1 plus cisplatin (SP) as adjuvant chemotherapy for stage III gastric cancer patients.

\section{Materials and Methods}

Stage III gastric cancer patients who had received curative gastrectomy with D2 lymphadenectomy were randomized into equal groups to receive adjuvant chemotherapy of eight cycles of DS (S-1 $70 \mathrm{mg} / \mathrm{m}^{2} /$ day on days $1-14$ plus docetaxel $35 \mathrm{mg} / \mathrm{m}^{2}$ on days 1 and 8) every 3 weeks or SP (S-1 $70 \mathrm{mg} / \mathrm{m}^{2} /$ day on days $1-14$ plus cisplatin $60 \mathrm{mg} / \mathrm{m}^{2}$ on day 1 ) every 3 weeks. The primary endpoint was 3-year disease-free survival (DFS) rate.

Results

Between November 2010 and July 2013, 153 patients ( 75 patients to DS and 78 patients to SP) were enrolled from 8 institutions in Korea. After the capecitabine plus oxaliplatin was approved based on the CLASSIC study, it was decided to close the study early. With a median follow-up duration of 56.9 months, the 3-year DFS rate between two groups was not significantly different (49.14\% in DS group vs. $52.5 \%$ in SP group). The most common grade $3-4$ adverse event was neutropenia (42.7\% in DS and $38.5 \%$ in SP, $p=0.351$ ). SP group had more grade $3-4$ anemia (1.3\% vs. $11.5 \%, p=0.037$ ), whereas grade $3-4$ hand-foot syndrome (4.1\% vs. $0 \%, p=0.025)$ and mucositis $(10.7 \%$ vs. $2.6 \%, p=0.001)$ were more common in DS group. Fifty-one patients (68\%) in DS group and 52 (66.7\%) in SP group finished planned treatment.

\section{Conclusion}

Our findings suggest that SP or DS is an effective and tolerable option for patients with curatively resected stage III gastric cancer.

\section{Key words}

Stomach neoplasms, Stage III, S-1 based doublet, Docetaxel, Cisplatin, Adjuvant chemotherapy
Correspondence: Sun Young Rha, MD, PhD Division of Medical Oncology, Department of Internal Medicine, Yonsei Cancer Center, Yonsei University College of Medicine, 50-1 Yonsei-ro, Seodaemun-gu, Seoul 03722, Korea Tel: 82-2-2228-8050

Fax: 82-2-362-5592

E-mail: rha7655@yuhs.ac

Received January 9, 2018

Accepted January 30, 2018

Published Online February 5, 2018

${ }^{*}$ Choong-kun Lee and Minkyu Jung contributed equally to this work. 


\section{Introduction}

Gastric cancer is the second leading cause of the cancer death worldwide, and is particularly prevalent in Eastern Asia, Eastern Europe, and South America [1]. The overall 5-year relative survival rate was $37.8 \%$ in the Surveillance, Epidemiology, and End Results data [2], and 55.7\% in the Korea National Cancer Incidence Database [3] for locally advanced gastric cancer. Complete surgical resection remains the only chance of a cure in gastric cancer patients, and gastrectomy with D2 lymphadenectomy is a standard surgical treatment for locally advanced gastric cancer. Since approximately $40 \%$ to $80 \%$ of gastric cancer patients still suffer recurrence and ultimately die from the disease [4], there has always been an urgent need for effective adjuvant chemotherapy or radiotherapy. The recent adjuvant trials in gastric cancer have shown improved survival in patients treated with adjuvant chemotherapy compared to those who underwent surgery alone [5,6] especially in Asia. Based on individual patient data from 17 randomized clinical trials, metaanalysis from the GASTRIC (Global Advanced/Adjuvant Stomach Tumor Research International Collaboration) group showed that postoperative adjuvant chemotherapy based on a fluorouracil regimen was associated with longer survival in gastric cancer compared with surgery alone [7]. In 2007, a large-scale study by the Adjuvant Chemotherapy Trial of S-1 for Gastric Cancer (ACTS-GC) group reported the first positive phase III result addressing superiority of adjuvant chemotherapy with an oral fluoropyrimidine, S-1 monotherapy versus surgery [5]. However, subgroup analysis revealed that S-1 only provided a survival benefit among patients with an early disease stage (II or IIIA) who had undergone D2 gastrectomy [8]. Therefore, a more efficient chemotherapy regimen, for example one based on a drug doublet, is needed to improve the outcome of patients with locally advancedespecially stage III-gastric cancer after curative resection. With only limited adjuvant S-1 monotherapy benefit in stage III gastric cancer patients even after 5-year follow up period of the ACTS-GC trial [8], and no general consensus about the best adjuvant treatment option, we designed a phase III trial to establish the effectiveness of an S-1 containing doublet regimen for advanced American Joint Committee on Cancer (AJCC) seventh stage III patients after curative gastrectomy. Capecitabine plus oxaliplatin seems to be effective for advanced stage II and III gastric cancer patients, but the high incidence of hand-foot syndrome and peripheral neuropathy are toxic for many patients [6]. And the CLASSIC trial result was not available when we planned this phase III trial. Because S-1 is tolerable and widely accepted as an effective therapeutic agent for resected gastric cancer in an adjuvant setting, adding other cytotoxic agent seemed reasonable.
In order to identify an efficient doublet antineoplastic regimen that could improve treatment outcome, among late stage advanced gastric cancer patients after D2 gastrectomy, the Post Operation chemotherapy with S-1 and Taxotere in curatively resected gastric cancer of stage III (POST) study was designed. This study compared the effect of two adjuvant S-1 based doublet regimens, S-1 plus docetaxel (DS) with $\mathrm{S}-1$ plus cisplatin (SP), on the disease-free survival (DFS) of D2 resected stage III gastric cancer patients. There have been several previous randomized control trials of DS or SP as palliative treatments in metastatic gastric cancer [9-11], but there has been no phase III study to directly compare these two doublet regimens, or to assess their use in adjuvant therapy for D2 resected stage III advanced gastric cancer.

\section{Materials and Methods}

\section{Study design and treatment}

The POST trial was an open-label, phase III, randomized controlled study, performed in eight centers in South Korea. The primary aim of the study was to compare the DFS following S-1 doublets between DS and SP. Gastrectomy with D2 lymphadenectomy has been performed as a standard procedure by all of the surgeons who participated in this trial and the pathology reports were standardized based on D2 dissection in each institute. The patients were randomly assigned to 6 months of treatment with eight cycles of either DS (intravenous docetaxel $\left[35 \mathrm{mg} / \mathrm{m}^{2}\right.$ on days 1 and 8 of each cycle] plus oral S-1 [35 mg/m² twice daily on days 1 to 14 of each cycle]) every 3 weeks, or SP (intravenous cisplatin [60 $\mathrm{mg} / \mathrm{m}^{2}$ on day 1] plus S-1 [same dosage utilized in DS]) every 3 weeks. Oral S-1 was ministered after meal within one hour. Pre-hydration before intravenous cisplatin following the institutional protocol was mandatory to prevent nephrotoxicity. The randomization was stratified by institution and disease stage (IIIA vs. IIIB vs. IIIC). Each stratum was randomized using the randomly permuted block method.

\section{Patients}

Inclusion criteria for patients were that they should have histopathologically confirmed AJCC seventh stage III gastric adenocarcinoma; undergone R0 resection (with no residual microscopic tumor cells including margin) with D2 lymph node dissection; age of 20 years or older; adequate renal function (creatinine clearance $>50 \mathrm{~mL} / \mathrm{min}$ ), hepatic function (total bilirubin $\leq 1.5$ times the upper limit of normal [ULN], aspartate or alanine aminotransferase $\leq 2.5$ times the ULN, 
alkaline phosphatase $\leq 2.5$ times the ULN), and hematological function (absolute neutrophil count $\geq 1.5 \times 10^{9} / \mathrm{L}$ or platelet count $\left.\geq 100 \times 10^{9} / \mathrm{L}\right)$; and had an Eastern Cooperative Oncology Group (ECOG) performance status of 0 or 1 . Exclusion criteria were as follows: a tumor other than adenocarcinoma; pregnancy or breast feeding; evidence of metastasis including peritoneal or distant metastasis; any previous treatment of cytotoxic chemotherapy, radiotherapy, or immunotherapy for gastric cancer; previous major surgery within 4 weeks before the start of the trial, or a failure to recover from the surgery; previous history of other malignancies within 5 years except for cured skin basal cell carcinoma or cured in situ cervix cancer; gastrointestinal obstruction or malabsorption syndrome that can negatively affect S-1 absorptions; R1 or R2 resection; other severe medical conditions; known dihydropyrimidine dehydrogenase deficiency; sensitivity to platinum agents or docetaxel; and inadequate organ function. All the patients had been randomized into either treatment group within 8 weeks after surgery.

\section{Study end points and assessments}

Three-year DFS rate was chosen as the primary endpoint because a recent meta-analysis showed that DFS is an acceptable surrogate end point for overall survival (OS) in trials of cytotoxic agents for gastric cancer in the adjuvant setting [12]. DFS was defined as the time from randomization to the time of recurrence of gastric cancer or death from any cause, and was to be analyzed by intent-to-treat. Secondary end points included OS, defined as the time from randomization to the time of death from any cause, and safety profiles (adverse events). Tumors were assessed radiographically using computed tomography at screening (baseline), every 3 months after randomization during years 1 and 2, every 6 months during year 3 , and then yearly.

Toxicity was closely monitored during each treatment cycle, and adverse events were graded according to the National Cancer Institute's Common Terminology Criteria for Adverse Events ver. 4.0. The dose of each chemotherapeutic agent could be reduced if any of the following toxicities occurred in the preceding cycle: grade 4 leukopenia, neutropenia or thrombocytopenia, febrile neutropenia grade 3 or higher, non-hematologic toxicity grade 3 or higher except for hand foot syndrome, cardiac toxicity, and elevated bilirubin of grade 2 or higher. The study drugs were discontinued if recovery did not occur within 3 weeks from the planned day 1 .

\section{Statistical methods}

The intent-to-treat (ITT) patient population, the population for all primary analyses, included all randomly assigned patients who had received at least one cycle of their allocated treatment. Three-year DFS rate, the primary end point, was predicted to be $40.0 \%$ and $55.0 \%$ in SP group and DS group, respectively (hazard ratio [HR], 0.65), based on two docetaxel and cisplatin combined studies [9,13]. Kaplan-Meier estimates and Cox regression analyses of DFS and OS were calculated. The two groups were compared using the stratified log-rank test. With a predicted median follow-up duration of 3 years, 173 events were necessary to ensure $80 \%$ power for a twosided test at a significance level of 0.05 . A sample size of 145 patients in each group was planned, allowing for a $10 \%$ dropout rate. The safety analysis included all the ITT patients. SPSS ver. 20 (IBM Corp., Armonk, NY) and the statistical software package $R$ ver. 13.0 were used for statistical analyses. A p-value of less than 0.05 was considered statistically significant.

\section{Accrual and time of analysis}

In January 2012, the phase III capecitabine and oxaliplatin adjuvant study in stomach cancer (CLASSIC) study showed a survival benefit of adjuvant capecitabine and oxaliplatin chemotherapy compared to surgery alone, among stage II-III gastric cancer patients who had undergone curative D2 gastrectomy [6]. Based on this trial, in March 2013, the Korean Food and Drug Administration approved the use of capecitabine and oxaliplatin for adjuvant chemotherapy after curative gastrectomy. Since patients could be reimbursed by National Insurance of Korea for the use of capecitabine and oxaliplatin as an adjuvant therapy, the accrual rate of the POST trial was reduced. The trial committee decided to close the study early with the last patient enrolled in July 2013, and the data cut-off date was April 30, 2017.

\section{Ethical statement}

The protocol was approved by the institutional review and ethics board of each participating center. The study was conducted in accordance with the Declaration of Helsinki and the Good Clinical Practice Guidelines defined by the International Conference on Harmonization. All patients provided written, informed consent before enrollment. The trial is registered at ClinicalTrials.gov (NCT01283217). 
Table 1. Baseline patients' characteristics

\begin{tabular}{|c|c|c|}
\hline Characteristic & DS ( $n=75)$ & $\mathrm{SP}(\mathrm{n}=78)$ \\
\hline \multicolumn{3}{|l|}{ Sex } \\
\hline Male & $46(61.3)$ & $56(71.8)$ \\
\hline Female & $29(38.7)$ & $22(28.2)$ \\
\hline Age, median (range, yr) & $54(33-74)$ & $58(25-72)$ \\
\hline \multicolumn{3}{|l|}{ ECOG performance } \\
\hline 0 & $45(60.0)$ & $53(67.9)$ \\
\hline 1 & $30(40.0)$ & $25(32.1)$ \\
\hline \multicolumn{3}{|l|}{ Cell type (WHO classification) } \\
\hline Tubular adenocarcinoma well differentiated & 0 & $2(0.3)$ \\
\hline Tubular adenocarcinoma moderately differentiated & $20(26.7)$ & $26(33.3)$ \\
\hline Tubular adenocarcinoma poorly differentiated & $34(45.2)$ & $35(44.9)$ \\
\hline Signet ring cell & $17(22.7)$ & $13(16.7)$ \\
\hline Hepatoid adenocarcinoma & $1(2.4)$ & 0 \\
\hline Mucinous adenocarcinoma & $2(2.7)$ & $1(2.4)$ \\
\hline Undifferentiated adenocarcinoma & $1(2.4)$ & $1(2.4)$ \\
\hline \multicolumn{3}{|l|}{ Lauren classification } \\
\hline Intestinal type & $11(14.7)$ & $16(20.5)$ \\
\hline Diffuse type & $29(38.7)$ & $18(23.1)$ \\
\hline Mixed & $2(2.7)$ & $8(10.3)$ \\
\hline Unknown & $33(44.0)$ & $36(45.2)$ \\
\hline No. of retrieved lymph nodes, median (interquartile range) & $43(34-55)$ & $40(29-53)$ \\
\hline \multicolumn{3}{|l|}{ Tumor stage } \\
\hline $\mathrm{T} 2$ & $2(2.7)$ & $3(3.8)$ \\
\hline T3 & $15(20.0)$ & $24(30.8)$ \\
\hline $\mathrm{T} 4$ & $58(77.3)$ & $51(65.4)$ \\
\hline \multicolumn{3}{|l|}{ Nodal stage } \\
\hline 0 & $1(1.3)$ & 0 \\
\hline 1 & $5(6.7)$ & $1(1.3)$ \\
\hline 2 & $23(30.7)$ & $24(30.8)$ \\
\hline 3 & $46(61.3)$ & $53(56.7)$ \\
\hline \multicolumn{3}{|l|}{ AJCC 7th stage } \\
\hline IIIA & $9(12.0)$ & $10(12.8)$ \\
\hline IIIB & $33(44.0)$ & $36(46.2)$ \\
\hline IIIC & $33(44.0)$ & $32(41.0)$ \\
\hline \multicolumn{3}{|l|}{ AJCC 6th stage } \\
\hline II & $4(5.3)$ & $6(7.7)$ \\
\hline IIIA & $26(34.7)$ & 31 (39.7) \\
\hline IIIB & $18(24.0)$ & $20(25.6)$ \\
\hline IV & $25(33.3)$ & $20(25.6)$ \\
\hline Duration from surgery to initiate chemotherapy, median (range, wk) & $6.13(2.7-8.7)$ & $5.66(2.5-7.8)$ \\
\hline
\end{tabular}

Values are presented as number $(\%)$ unless otherwise indicated. DS, docetaxel plus S-1; SP, S-1 plus cisplatin; ECOG, Eastern Cooperative Oncology Group; WHO, World Health Organization; AJCC, American Joint Committee on Cancer. 

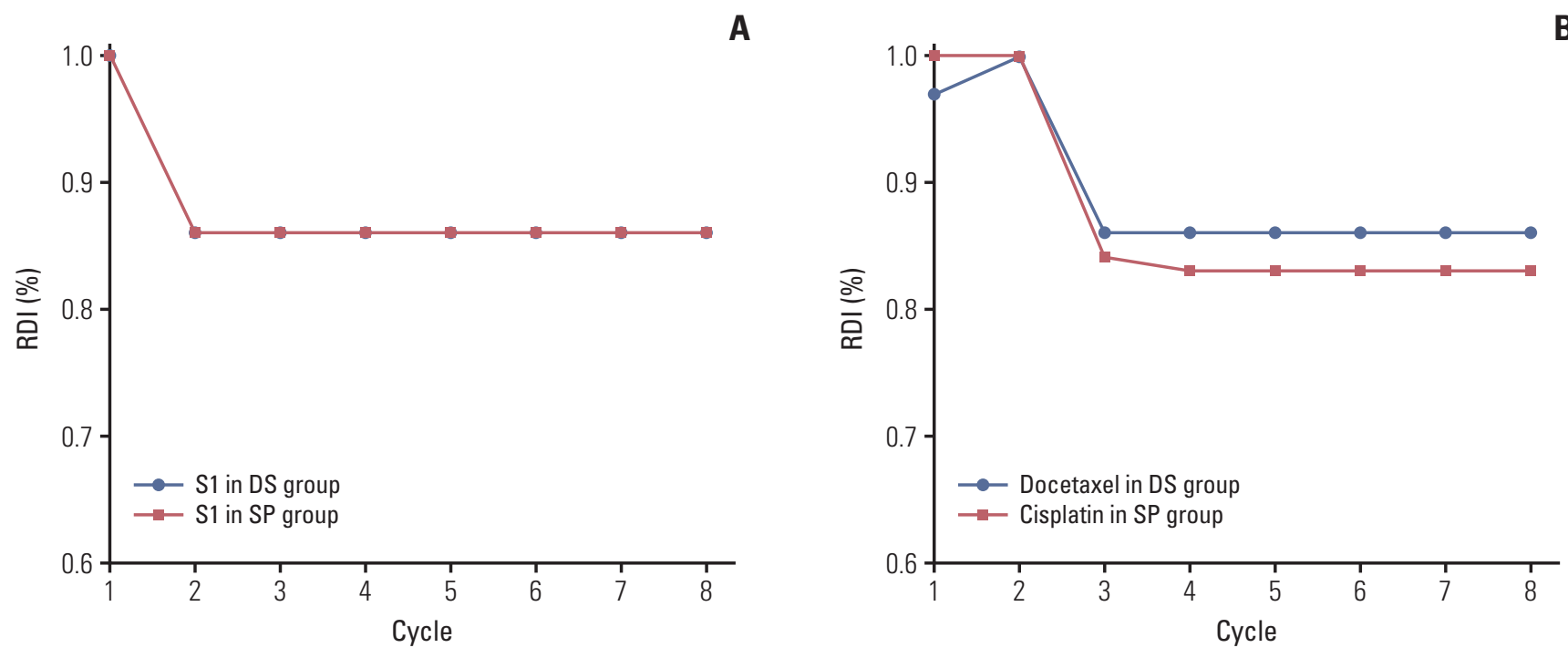

Fig. 1. Median relative dose intensities per regimen and cycle. Median relative dose intensities (RDIs) of S-1 in both groups (A) and RDIs of docetaxel in docetaxel plus S-1 (DS) group or cisplatin in S-1 plus cisplatin (SP) group (B).

Table 2. Adverse events $(n=153)$

\begin{tabular}{|c|c|c|c|c|c|}
\hline \multirow{2}{*}{ Treatment-related adverse event } & \multicolumn{2}{|c|}{ DS $(n=75)$} & \multicolumn{2}{|c|}{$\mathrm{SP}(\mathrm{n}=78)$} & \multirow{2}{*}{ p-value } \\
\hline & All & G3/4 & All & G3/4 & \\
\hline Any & $74(98.7)$ & $46(61.3)$ & 77 (98.7) & $44(56.4)$ & 0.536 \\
\hline \multicolumn{6}{|l|}{ Hematologic toxicity } \\
\hline Febrile neutropenia & $3(4.0)$ & $2(2.7)$ & $3(3.8)$ & $3(3.8)$ & 0.548 \\
\hline Neutropenia & $51(68.0)$ & $32(42.7)$ & $28(35.9)$ & $30(38.5)$ & 0.351 \\
\hline Anemia & $41(54.0)$ & $1(1.3)$ & $45(57.6)$ & $9(11.5)$ & 0.037 \\
\hline Thrombocytopenia & $2(2.6)$ & $1(1.3)$ & $19(24.3)$ & $1(1.3)$ & $<0.001$ \\
\hline \multicolumn{6}{|l|}{ Non-hematologic toxicity } \\
\hline Abdominal pain & $24(32.0)$ & $3(4.1)$ & $15(19.2)$ & $2(2.6)$ & 0.193 \\
\hline Anorexia & $48(64.0)$ & $5(6.7)$ & $47(60.2)$ & $5(6.4)$ & 0.892 \\
\hline Nausea & $49(65.3)$ & $2(2.7)$ & $51(65.4)$ & $2(2.6)$ & 0.999 \\
\hline Vomiting & $16(21.3)$ & $1(1.3)$ & $17(21.8)$ & $1(1.3)$ & 0.997 \\
\hline Hand foot syndrome & $18(24.0)$ & $3(4.1)$ & $7(9.0)$ & 0 & 0.025 \\
\hline Mucositis & $39(52.0)$ & $8(10.7)$ & $18(23.1)$ & $2(2.6)$ & 0.001 \\
\hline Diarrhea & $40(53.3)$ & $2(2.7)$ & $35(44.9)$ & $3(3.8)$ & 0.478 \\
\hline Paronychia & $8(10.7)$ & 0 & 0 & 0 & 0.003 \\
\hline Alopecia & $34(45.3)$ & 0 & $4(5.1)$ & 0 & $<0.001$ \\
\hline Fatigue & $12(16.0)$ & $1(1.3)$ & $14(18.0)$ & $1(1.3)$ & 0.944 \\
\hline General weakness & $16(21.3)$ & $1(1.3)$ & $23(29.5)$ & $2(2.6)$ & 0.493 \\
\hline Neuropathy & $17(22.7)$ & 0 & 15 (19.2) & $1(1.3)$ & 0.487 \\
\hline
\end{tabular}

Values are presented as number (\%). One treatment related death in DS group: thromboembolism event. DS, docetaxel plus S-1; SP, S-1 plus cisplatin. 


\section{Results}

\section{Patient characteristics}

Between November 2010 and July 2013, a total of 153 patients from eight centers in South Korea were randomly assigned to DS ( $\mathrm{n}=75)$ or SP ( $\mathrm{n}=78)$ treatment groups (S1 Fig.). Patient demographics and baseline disease characteristics were well balanced between the two groups. Poorly differentiated and diffuse types of adenocarcinoma were the most common histological type. Most of the patients had AJCC seventh edition stage IIIB $(n=69,45.1 \%)$ or stage IIIC disease $(\mathrm{n}=65,42.5 \%)($ Table 1$)$.

\section{Treatment compliance and toxicity}

The median number of treatment cycles in each group was
8 (range, 1 to 8 cycles). The median relative dose intensity (RDI) was 0.86 for both docetaxel and S-1 in DS group and 0.83 for cisplatin and 0.86 for S-1 in SP group. The RDI of S-1 in both groups was reduced from the second cycle, and the RDI of docetaxel in DS group and cisplatin in SP group was reduced from the third cycle (Fig. 1). The number of the patients who could not complete the full eight cycles of chemotherapy was $24(32.0 \%)$ in DS group and $26(33.3 \%)$ in SP group ( $\mathrm{p}=0.683)$.

Most patients $(98.7 \%)$ reported at least one adverse event. The total incidence of grade 3 or 4 adverse events was 46 $(61.3 \%)$ in DS group and $44(56.4 \%)$ in SP group $(p=0.536)$. The most common grade 3 or 4 adverse event was neutropenia $(42.7 \%$ in DS group and $38.5 \%$ in SP group, $\mathrm{p}=0.351$ ) (Table 2). SP treatment was associated with more frequent hematologic adverse events such as grade 3 or 4 anemia $(1.3 \%$ vs.11.5\%, $\mathrm{p}=0.037)$ or any grade of thrombocytopenia ( $2.6 \%$ vs. $24.3 \%, \mathrm{p}<0.001)$. However, grade 3 or 4 non-hema-

Table 3. The reasons for chemotherapy cycle delays and reductions

\begin{tabular}{|c|c|c|c|c|}
\hline & \multicolumn{2}{|c|}{ Delayed cycles } & \multicolumn{2}{|c|}{ Dose reduced cycles } \\
\hline & DS $(n=495)$ & SP $(n=512)$ & DS $(n=495)$ & $\mathrm{SP}(\mathrm{n}=512)$ \\
\hline Hematologic & 84 & 152 & 35 & 33 \\
\hline Neutropenia & 83 & 149 & 35 & 31 \\
\hline Febrile neutropenia & 1 & 1 & 0 & 1 \\
\hline Anemia & 0 & 1 & 0 & 0 \\
\hline Thrombocytopenia & 0 & 1 & 0 & 1 \\
\hline Non-hematologic & 46 & 20 & 36 & 23 \\
\hline General weakness & 8 & 2 & 4 & 4 \\
\hline Nausea/Vomiting & 5 & 1 & 5 & 4 \\
\hline Patients' refusal & 4 & 6 & 2 & 0 \\
\hline Mucositis & 4 & 2 & 9 & 2 \\
\hline Urticaria/Skin rash & 4 & 0 & 1 & 0 \\
\hline Anorexia & 3 & 4 & 3 & 3 \\
\hline Hand-foot syndrome & 3 & 0 & 4 & 0 \\
\hline Weight loss & 3 & 0 & 1 & 3 \\
\hline Fatigue & 0 & 3 & 3 & 1 \\
\hline Diarrhea & 0 & 2 & 1 & 3 \\
\hline Hemorrhoid & 2 & 0 & 0 & 0 \\
\hline Nail Infection & 2 & 0 & 1 & 0 \\
\hline Abdominal pain & 1 & 0 & 0 & 2 \\
\hline Generalized edema & 2 & 0 & 0 & 0 \\
\hline Common cold & 2 & 0 & 0 & 0 \\
\hline Herpes zoster & 1 & 0 & 1 & 0 \\
\hline Intestinal obstruction & 1 & 0 & 0 & 0 \\
\hline Bilirubin elevation & 1 & 0 & 1 & 0 \\
\hline Dizziness & 0 & 0 & 0 & 1 \\
\hline Total, n (\%) & $130(26.3)$ & $172(33.6)$ & $71(14.3)$ & $56(10.9)$ \\
\hline
\end{tabular}

DS, docetaxel plus S-1; SP, S-1 plus cisplatin. 

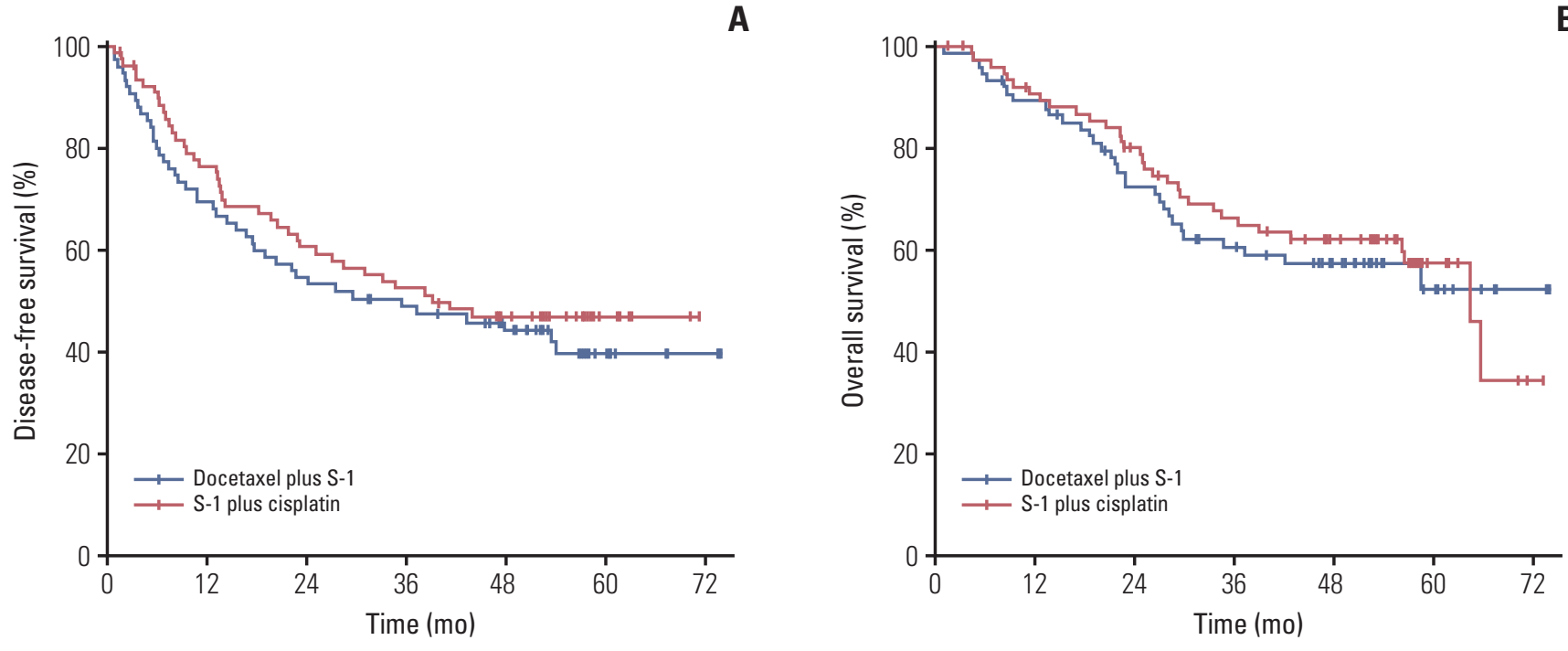

Fig. 2. Kaplan-Meier curves of disease-free survival (A) and overall survival (B) in the intention-to-treat population.

tologic adverse events including hand-foot syndrome $(4.1 \%$ vs. $0 \%, \mathrm{p}=0.025)$ and mucositis $(10.7 \%$ vs. $2.6 \%, \mathrm{p}=0.001)$ were more commonly observed in DS group. Grade 1 or 2 paronychia $(10.7 \%$ vs. $0 \%, \mathrm{p}=0.003)$ and alopecia $(45.3 \%$ vs. $5.1 \%, \mathrm{p}<0.001)$ were also more common in DS group. There was one treatment-related death in DS group, from a thromboembolic event. The day after starting the seventh cycle of DS treatment, this 67-year-old female patient's dyspnea on exertion worsened, and the patient died due to left pulmonary artery thromboembolism. The patient did not have any other pre-disposing factor for pulmonary thromboembolism.

Among total 495 treatment cycles for DS group and 512 cycles for SP group, 130 cycles $(26.3 \%)$ in DS group and 172 cycles $(33.6 \%)$ in SP group ( $p=0.011$ ) were delayed. The chemotherapy dose was reduced in 71 cycles $(14.3 \%)$ in DS group and 56 cycles $(10.9 \%)$ in SP group $(p=0.104)$. Overall, the most common reason for delay in the initiation of treatment in each cycle and for the dose reduction was neutropenia $(76.8 \%$ for of delayed cycles and $52.0 \%$ of dose reduced cycles) (Table 3). With respect to adverse events (Table 2), treatment delays were more common in SP group which was associated with more frequent hematologic toxicities, whether dose reductions were more common in DS group which experienced more non-hematologic toxicities.

The treatment discontinuations were permanently developed from 24 patients in DS group and from 26 patients in SP groups. The main reason for discontinued treatment was toxicity (12 [50.0\%] in DS group and 13 [50.0\%] in SP group) (S1 Fig.). The reasons for discontinuation due to toxicity in
DS group were allergic reaction $(n=3)$, neutropenia, afferent loop syndrome, skin rash, thromboembolic event, ileus, hand-foot syndrome, general weakness, dyspnea, and nausea/vomiting ( $n=1$ for each). In SP group, toxicities that resulted in treatment discontinuation were neutropenia $(n=3)$, general weakness $(n=2)$, thrombocytopenia, myocardiac infarction, neuropathy, skin rash, anorexia, acute cerebral infarction and pneumonitis ( $\mathrm{n}=1$ for each). Three patients in DS group and four patients in SP group were off the study because of gastric cancer recurrence during the course of the trial treatment.

\section{Survival analysis}

The median duration of follow-up was 53.6 months (range, 1.1 to 74.0 months) for DS group and 57.3 months (range, 1.7 to 73.1 months) for SP group. At the cut-off date for data collection on April 30, 2017, 43 patients (57.3\%) in DS group and 40 patients $(53.3 \%)$ in SP group developed DFS events. The most common site of recurrence was the peritoneum (20 and 11 cases in DS and SP groups, respectively) (Table comparing pattern of recurrences in S2 Table). Primary endpoint, the 3-year DFS rate, was $49.1 \%$ in DS group (median DFS, 35.5 months) and 52.5\% in SP group (median DFS, 39.3 months). The 3-year survival rate was $60.7 \%$ among DS group and $66.4 \%$ among SP group, with $31(41.3 \%)$ and $32(41.0 \%)$ deaths in DS group (median OS, not reached) and SP group (median OS, 64.4 months), during the follow-up period. Every death was cancer-related. There was no statistically significant difference in DFS (HR, 1.201; 95\% confidence 


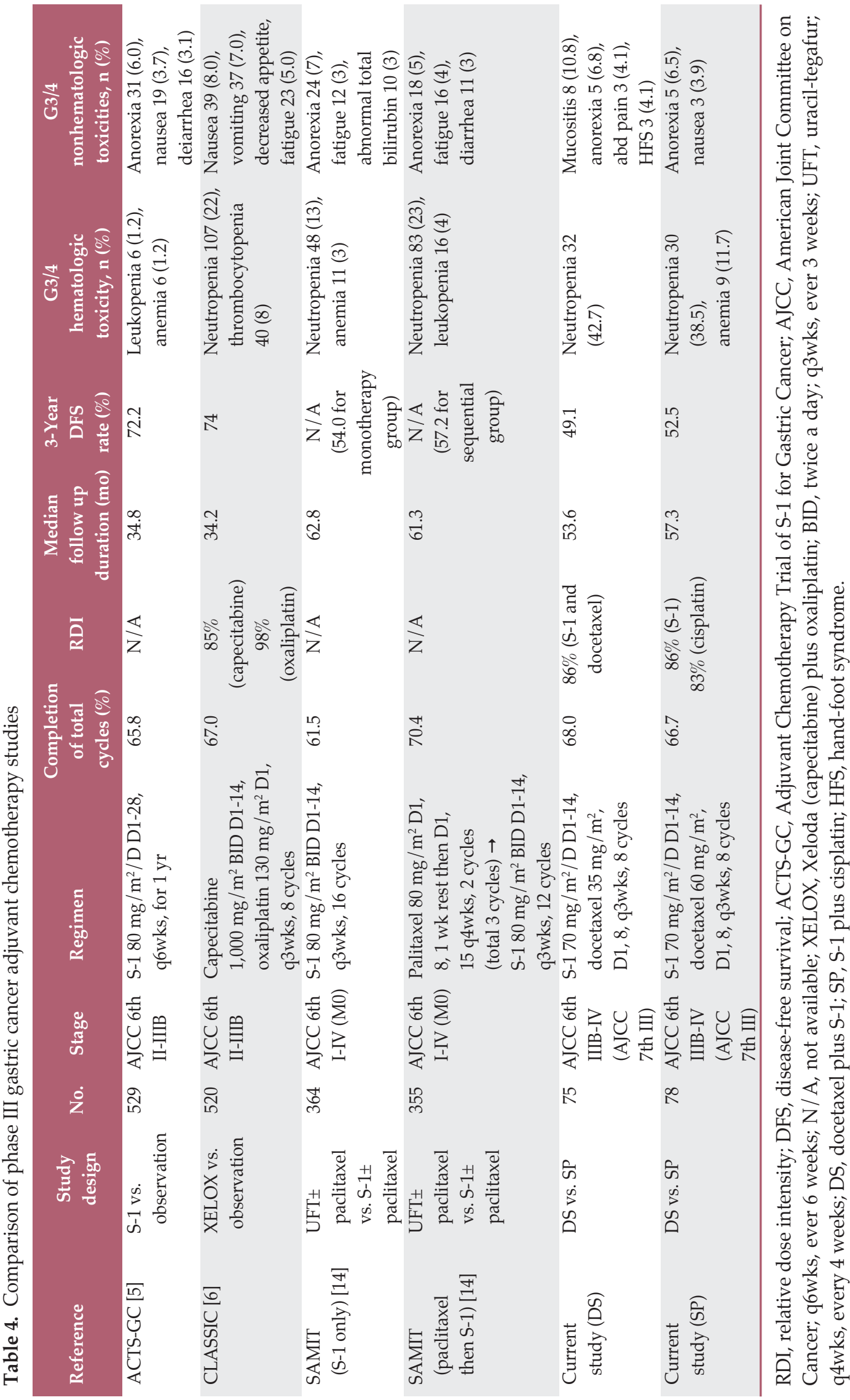


interval [CI], 0.781 to $1.848 ; \mathrm{p}=0.404)$ or OS (HR, $1.081 ; 95 \%$ CI, 0.659 to $1.772 ; \mathrm{p}=0.757$ ) between DS and SP groups (Fig. 2). The DFS and OS of eligible patients were also analyzed according to sex, age, ECOG performance status, cancer stage based on AJCC seventh, tumor stage, and nodal stage. Stage IIIC subgroup among SP group showed significant benefit of DFS (HR, 2.018; 95\% CI, 1.108 to 3.675; $\mathrm{p}=0.022$ ), and OS (HR, 2.149; 95\% CI, 1.018 to $4.533 ; \mathrm{p}=0.048$ ), but there was no significant interaction between the treatment group and any other subgroups in terms of survival (Forest plots in S3 Fig.).

\section{Discussion}

This POST trial is the first randomized phase III trial comparing efficacy and tolerability of two S-1 based doublet regimens, DS and SP, in curatively resected stage III gastric cancer patients based on the AJCC seventh edition. Unfortunately, because of the early termination, we could not show any statistically significant advantage in terms of OS or DFS for either regimen. However, both treatments seem to be an effective and tolerable option for these patients.

S-1 was regarded to have advantages over capecitabine among Asians in terms of reducing incidence of toxicities such as stomatitis and hand-foot syndrome [15]. Several chemotherapeutic regimens combining S-1 with other anticancer agents have been shown to improve the response rate or median survival time in gastric cancer, mostly in phase II studies [16-20]. From among many possible combinations of anti-cancer drugs, we compared S-1 plus taxane (DS) with S-1 plus platinum (SP) in this phase III study. Taxanes like docetaxel has been shown to prolong survival when used as a palliative treatment for advanced gastric cancer [21]. The phase III START trial showed that the combination of S-1 and docetaxel is more effective than S-1 monotherapy for metastatic gastric cancer patients, with longer survival (12.5 months vs. 10.8 months, $\mathrm{p}=0.032)$ and a significant $(16 \%)$ reduction in the risk of death [22]. In addition, a phase II study comparing docetaxel plus S-1 to docetaxel plus cisplatin suggested that DS was more effective (OS, 16 vs. 8.3 months) among chemotherapy naïve stage IV advanced gastric cancer patients [9]. Recently, another large phase III adjuvant trial (SAMIT) result was published [14]. The study tried to assess the superiority of adding paclitaxel to oral fluoropyrimidines as sequential treatment to locally advanced gastric cancer patients after D2 dissection, but the trial failed to show survival improvement. Cisplatin is generally accepted as a benchmark treatment when combined with 5-fluorouracil for advanced gastric cancer, although it has several drawbacks, including high incidences of nausea, vomiting [23] and renal toxicity [24]. Cisplatin has also been shown to have an additive effect when combined with S-1 in advanced gastric cancer. In the phase III SPIRIT trial, SP resulted in significantly longer survival compared to S-1 alone (OS, 13 months vs. 11 months; $\mathrm{p}=0.04$ ) and a better response rate (54\% vs. 31\%) [25]. Based on these previous studies, the aim of this study was compare two S-1 based doublets to determine whether there was a significant increase in the 3-year DFS rate when DS or SP were used to treat patients with stage III gastric cancer.

The addition of docetaxel or cisplatin to S-1 was well-tolerated. We compared compliances and toxicities of our study (POST) with three published phase III studies of adjuvant chemotherapy for gastric cancer; ACTS-GC, CLASSIC, and SAMIT (Table 4) $[5,6,14]$. Sixty-eight percent of patients in DS group and $66.7 \%$ of patients in SP group completed 8 cycles as planned, which was comparable to that achieved in the ACTS-GC (65.8\% for S- 1 monotherapy), SAMIT (61.5\% for S-1 monotherapy or 70.4\% for paclitaxel then S-1 sequential therapy), and CLASSIC (67\% for capecitabine plus oxaliplatin) trials. The median RDI was $86 \%$ for S- 1 in both groups, $86 \%$ for docetaxel in DS group, and $83 \%$ for cisplatin in SP group. These findings were also comparable to those of CLASSIC trial (capecitabine $85 \%$ and oxaliplatin 98\%). Comparing toxicities among patients treated using DS or SP with those who were administered S-1 monotherapy (ACTSGC and SAMIT) revealed that adding docetaxel or cisplatin increased hematologic toxicity (neutropenia and anemia) but did not increased non-hematologic toxicities. In the capecitabine plus oxaliplatin group of the CLASSIC trial, $56 \%$ of patients experienced grade 3 or 4 adverse events, while in our study $61.3 \%$ and $56.4 \%$ of patients in DS and SP groups, respectively. A comparison of the treatment groups in this study revealed that patients treated with DS more frequently had non-hematologic toxicities, while those treated with SP more frequently suffered from hematologic toxicities (Table 2). These higher incidences of non-hematologic adverse events in DS group was related to more frequent dose reduction, and higher incidences of hematologic adverse events observed in SP group was related to more frequent treatment cycle delays (Table 3). S4 Table shows a comparison of compliancy rates and toxicities in previously reported adjuvant DS or SP chemotherapy trials, excluding those in which S-1 was given as a monotherapy during the first cycle [26-30].

The relative small number of accrued patients reduced the statistical power of our study. It is hard to compare survival outcomes of our study to that of previous studies, because this study only included stage III gastric cancer patients diagnosed on the basis of the AJCC seventh edition, and the previous adjuvant phase III studies included patients with stage II or III gastric cancer based on the AJCC sixth edition 
$[6,8]$. The more recent staging system classifies more than 15 positive nodes (N3b) as stage IIB or III, whereas it would have been classified as stage IV by the AJCC sixth edition staging system. Furthermore, invasion of the adjacent structure (T4b in the AJCC seventh edition and T4 in the AJCC sixth edition) with any positive node was classed as stage IV disease in the AJCC sixth edition but stage IIIB or IIIC disease in the AJCC seventh edition (S5 Fig.). Comparing the distribution of disease stages according to the AJCC seventh and sixth editions showed that 45 patients ( 3 patients with stage IIIA, 15 patients with stage IIIB, and 27 patients with stage IIIC; $29.4 \%$ in total) in this study would have been stage IV according to the AJCC sixth edition, and would thus not be eligible for the previous ACTS-GC or CLASSIC trials (S6 Fig.). So it is difficult to compare precisely, but our study has enrolled higher stage patients, and survival is similar when compared to Stage IIIB patients of CLASSIC or ACTS-GC trials.

In conclusion, postoperative DS and SP regimens were well tolerated, and the safety data presented here are comparable with that of previous studies. Since the trial was closed early, it didn't have sufficient statistical power to show a survival advantage for either regimen. We believe that our preliminary findings warrant further clinical investigation.

\section{Electronic Supplementary Material}

Supplementary materials are available at Cancer Research and Treatment website (https:// www.e-crt.org).

\section{Conflicts of Interest}

The drugs were supplied by Sanofi-Aventis Korea and Jeil Pharmaceutical.

\section{Acknowledgments}

We thank the patients, their families, all the investigators, subinvestigators, nurses and study teams. This research was supported by the Public Welfare \& Safety Research Program through the National Research Foundation of Korea (NRF), funded by the Ministry of Science, ICT \& Future Planning (2010-0020841).

\section{Author Details}

${ }^{1}$ Division of Medical Oncology, Department of Internal Medicine, Yonsei Cancer Center, Yonsei University College of Medicine, Seoul, ${ }^{2}$ Department of Biostatistics, Yonsei University College of Medicine, Seoul, ${ }^{3}$ Division of Hematology and Oncology, Department of Internal Medicine, Gachon University Gil Hospital, Incheon, ${ }^{4}$ Department of Hematology-Oncology, Ajou University School of Medicine, Suwon, ${ }^{5}$ Division of Hemato-Oncology, Department of Internal Medicine, Hallym University Medical Center, Hallym University College of Medicine, Anyang, ${ }^{6}$ Division of HematologyOncology, Department of Internal Medicine, Inje University Busan Paik Hospital, Inje University College of Medicine, Busan, 'Division of Hematology-Oncology, Department of Internal Medicine, Inha University School of Medicine, Incheon, ${ }^{8}$ Division of HematoOncology, Department of Internal Medicine, Veterans Health Service Medical Center, Seoul, ' Division of Hematology-Oncology, Department of Internal Medicine, Yeungnam University College of Medicine, Daegu, ${ }^{10}$ Department of Surgery, Yonsei Cancer Center, Yonsei University College of Medicine, Seoul, ${ }^{11}$ Song-Dang Institutefor Cancer Research, Yonsei University College of Medicine, Seoul, ${ }^{12}$ Brain Korea 21 Project for Medical Science, Yonsei University College of Medicine, Seoul, Korea

\section{References}

1. Jemal A, Bray F, Center MM, Ferlay J, Ward E, Forman D. Global cancer statistics. CA Cancer J Clin. 2011;61:69-90.

2. Seyedin S, Wang PC, Zhang Q, Lee P. Benefit of adjuvant chemoradiotherapy for gastric adenocarcinoma: a SEER population analysis. Gastrointest Cancer Res. 2014;7:82-90.

3. Jung KW, Won YJ, Kong HJ, Oh CM, Shin A, Lee JS. Survival of korean adult cancer patients by stage at diagnosis, 20062010: national cancer registry study. Cancer Res Treat. 2013;45: 162-71.

4. Gallo A, Cha C. Updates on esophageal and gastric cancers. World J Gastroenterol. 2006;12:3237-42.

5. Sakuramoto S, Sasako M, Yamaguchi T, Kinoshita T, Fujii M,
Nashimoto A, et al. Adjuvant chemotherapy for gastric cancer with S-1, an oral fluoropyrimidine. N Engl J Med. 2007;357: 1810-20.

6. Bang YJ, Kim YW, Yang HK, Chung HC, Park YK, Lee KH, et al. Adjuvant capecitabine and oxaliplatin for gastric cancer after D2 gastrectomy (CLASSIC): a phase 3 open-label, randomised controlled trial. Lancet. 2012;379:315-21.

7. GASTRIC (Global Advanced/Adjuvant Stomach Tumor Research International Collaboration) Group, Paoletti X, Oba K, Burzykowski T, Michiels S, Ohashi Y, et al. Benefit of adjuvant chemotherapy for resec-table gastric cancer: a metaanalysis. JAMA. 2010;303:1729-37. 
8. Sasako M, Sakuramoto S, Katai H, Kinoshita T, Furukawa H, Yamaguchi T, et al. Five-year outcomes of a randomized phase III trial comparing adjuvant chemotherapy with S-1 versus surgery alone in stage II or III gastric cancer. J Clin Oncol. 2011;29:4387-93.

9. Jeung HC, Rha SY, Im CK, Shin SJ, Ahn JB, Yang WI, et al. A randomized phase 2 study of docetaxel and S-1 versus docetaxel and cisplatin in advanced gastric cancer with an evaluation of SPARC expression for personalized therapy. Cancer. 2011;117:2050-7.

10. Tsuburaya A, Morita S, Kodera Y, Kobayashi M, Shitara K, Yamaguchi $\mathrm{K}$, et al. A randomized phase II trial to elucidate the efficacy of capecitabine plus cisplatin (XP) and S-1 plus cisplatin (SP) as a first-line treatment for advanced gastric cancer: XP ascertainment vs. SP randomized PII trial (XParTS II). BMC Cancer. 2012;12:307.

11. Mochiki E, Ogata K, Ohno T, Toyomasu Y, Haga N, Fukai Y, et al. Phase II multi-institutional prospective randomised trial comparing S-1+paclitaxel with S-1+cisplatin in patients with unresectable and/or recurrent advanced gastric cancer. Br J Cancer. 2012;107:31-6.

12. Oba K, Paoletti X, Alberts S, Bang YJ, Benedetti J, Bleiberg H, et al. Disease-free survival as a surrogate for overall survival in adjuvant trials of gastric cancer: a meta-analysis. J Natl Cancer Inst. 2013;105:1600-7.

13. Ajani JA, Moiseyenko VM, Tjulandin S, Majlis A, Constenla M, Boni C, et al. Clinical benefit with docetaxel plus fluorouracil and cisplatin compared with cisplatin and fluorouracil in a phase III trial of advanced gastric or gastroesophageal cancer adenocarcinoma: the V-325 Study Group. J Clin Oncol. 2007;25:3205-9.

14. Tsuburaya A, Yoshida K, Kobayashi M, Yoshino S, Takahashi M, Takiguchi N, et al. Sequential paclitaxel followed by tegafur and uracil (UFT) or S-1 versus UFT or S-1 monotherapy as adjuvant chemotherapy for $\mathrm{T} 4 \mathrm{a} / \mathrm{b}$ gastric cancer (SAMIT): a phase 3 factorial randomised controlled trial. Lancet Oncol. 2014;15:886-93.

15. Lee JL, Kang YK, Kang HJ, Lee KH, Zang DY, Ryoo BY, et al. A randomised multicentre phase II trial of capecitabine vs S-1 as first-line treatment in elderly patients with metastatic or recurrent unresectable gastric cancer. Br J Cancer. 2008;99: 584-90.

16. Koizumi W, Tanabe S, Saigenji K, Ohtsu A, Boku N, Nagashima F, et al. Phase I/II study of S-1 combined with cisplatin in patients with advanced gastric cancer. Br J Cancer. 2003;89:2207-12.

17. Yoshida K, Ninomiya M, Takakura N, Hirabayashi N, Takiyama W, Sato Y, et al. Phase II study of docetaxel and S-1 combination therapy for advanced or recurrent gastric cancer. Clin Cancer Res. 2006;12(11 Pt 1):3402-7.

18. Kakeji Y, Oki E, Egashira A, Sadanaga N, Takahashi I, Morita
M, et al. Phase II study of biweekly docetaxel and S-1 combination therapy for advanced or recurrent gastric cancer. Oncology. 2009;77:49-52.

19. Mochiki E, Ohno T, Kamiyama Y, Aihara R, Haga N, Ojima $\mathrm{H}$, et al. Phase I/ II study of S-1 combined with paclitaxel in patients with unresectable and/or recurrent advanced gastric cancer. Br J Cancer. 2006;95:1642-7.

20. Inokuchi M, Yamashita T, Yamada H, Kojima K, Ichikawa W, Nihei Z, et al. Phase I/ II study of S-1 combined with irinotecan for metastatic advanced gastric cancer. Br J Cancer. 2006;94: 1130-5.

21. Van Cutsem E, Moiseyenko VM, Tjulandin S, Majlis A, Constenla M, Boni C, et al. Phase III study of docetaxel and cisplatin plus fluorouracil compared with cisplatin and fluorouracil as first-line therapy for advanced gastric cancer: a report of the V325 Study Group. J Clin Oncol. 2006;24:4991-7.

22. Koizumi W, Kim YH, Fujii M, Kim HK, Imamura H, Lee KH, et al. Addition of docetaxel to S-1 without platinum prolongs survival of patients with advanced gastric cancer: a randomized study (START). J Cancer Res Clin Oncol. 2014;140:319-28.

23. American Society of Clinical Oncology, Kris MG, Hesketh PJ, Somerfield MR, Feyer P, Clark-Snow R, et al. American Society of Clinical Oncology guideline for antiemetics in oncology: update 2006. J Clin Oncol. 2006;24:2932-47.

24. Arany I, Safirstein RL. Cisplatin nephrotoxicity. Semin Nephrol. 2003;23:460-4.

25. Koizumi W, Narahara H, Hara T, Takagane A, Akiya T, Takagi M, et al. S-1 plus cisplatin versus S-1 alone for first-line treatment of advanced gastric cancer (SPIRITS trial): a phase III trial. Lancet Oncol. 2008;9:215-21.

26. Tamura S, Fujitani K, Kimura Y, Tsuji T, Matsuyama J, Iijima $S$, et al. Phase II feasibility study of adjuvant S-1 plus docetaxel for stage III gastric cancer patients after curative D2 gastrectomy. Oncology. 2011;80:296-300.

27. Fujitani K, Tamura S, Kimura Y, Tsuji T, Matsuyama J, Iijima $S$, et al. Three-year outcomes of a phase II study of adjuvant chemotherapy with S-1 plus docetaxel for stage III gastric cancer after curative D2 gastrectomy. Gastric Cancer. 2014;17:34853.

28. Lee SS, Jeung HC, Chung HC, Noh SH, Hyung WJ, Ahn JY, et al. A pilot study of S-1 plus cisplatin versus 5-fluorouracil plus cisplatin for postoperative chemotherapy in histological stage IIIB-IV (M0) gastric cancer. Invest New Drugs. 2012;30:357-63.

29. Kang BW, Kim JG, Chae YS, Lee YJ, Lee SJ, Moon JH, et al. Pilot study of adjuvant chemotherapy with 3-week combination of S-1 and cisplatin for patients with stage II-IV (M0) gastric cancer. Invest New Drugs. 2012;30:1671-5.

30. Fujii M. Chemotherapy for advanced gastric cancer: ongoing phase III study of S-1 alone versus S-1 and docetaxel combination (JACCRO GC03 study). Int J Clin Oncol. 2008;13:201-5. 